\title{
Erratum: First-principles study of possible shallow donors in $\mathrm{ZnAl}_{2} \mathrm{O}_{4}$ spinel [Phys. Rev. B 87, 174101 (2013)]
}

\author{
H. Dixit, Nandan Tandon, S. Cottenier, R. Saniz, D. Lamoen, and B. Partoens \\ (Received 5 August 2013; published 26 August 2013)
}

DOI: 10.1103/PhysRevB.88.059905

PACS number(s): 71.10.-w, 71.15.-m, 71.20.Nr, 99.10.Cd

In our recent paper on possible shallow donors in $\mathrm{ZnAl}_{2} \mathrm{O}_{4}$ spinel, the defect formation energies of the native defects for different charge states were calculated using the GGA and hybrid HSE06 functional. The results were presented in Figs. 5 and 6, respectively, of the paper. However, due to a typographic error in the calculation of the chemical potentials, the figures have to be replaced by Figs. 5 and 6 below. The difficulty of the GGA functional to properly describe defect states remains essentially the same, e.g., the $\mathrm{O}$ vacancy is still predicted to be an amphoteric defect. Focusing on the conclusions regarding the HSE06 results (see Fig. 6), one can see that the antisite defect $\mathrm{Al}_{\mathrm{Zn}}$ is still a shallow donor and the oxygen vacancy is still a deep donor. The other native defects are of the amphoteric type. The formation energy of the antisite defect $\mathrm{Al}_{\mathrm{Zn}}$ has increased. In oxygen-rich conditions, it is still the donor with the lowest formation energy. However, under Zn-rich conditions, the oxygen vacancy has the lowest formation energy of all donors for Fermi values close to the conduction band minimum. Furthermore, due to the large band gap of $\mathrm{ZnAl}_{2} \mathrm{O}_{4}$ and

Zn rich $(G G A)$

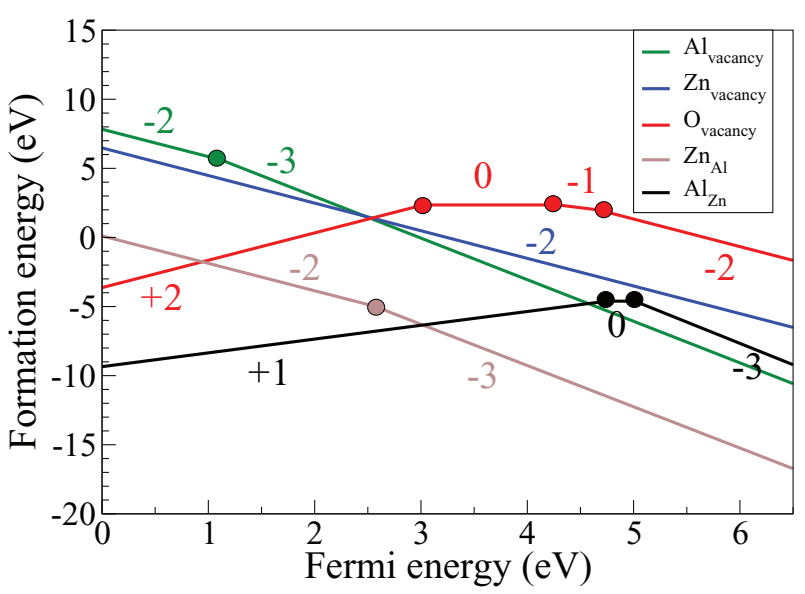

$\mathrm{O}$ rich $(\mathrm{GGA})$

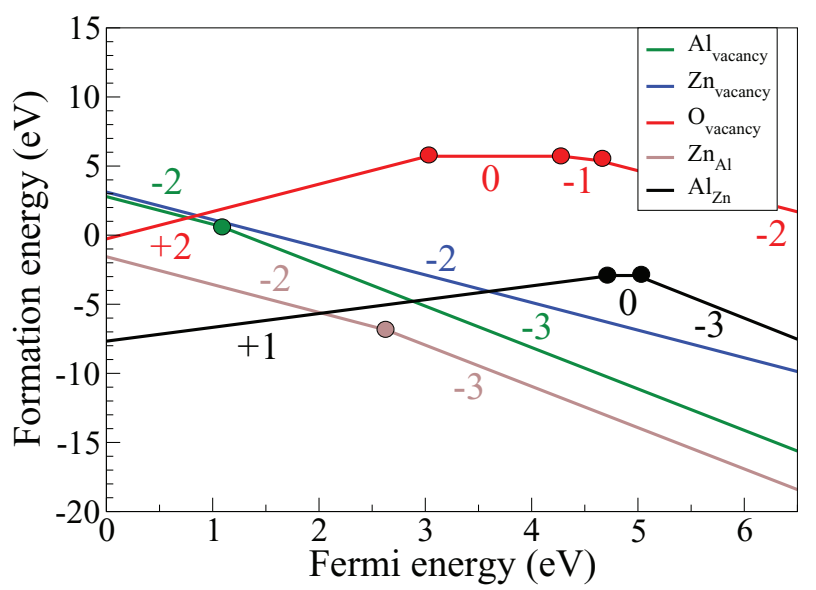

FIG. 5. (Color online) Calculated intrinsic defect formation energies as a function of the Fermi energy under Zn- and O-rich conditions with the GGA. The zero of the Fermi level corresponds to the valence band maximum, and the conduction band minimum is set at the GW band gap of $6.55 \mathrm{eV}$.

Zn rich (Hybrid functional)

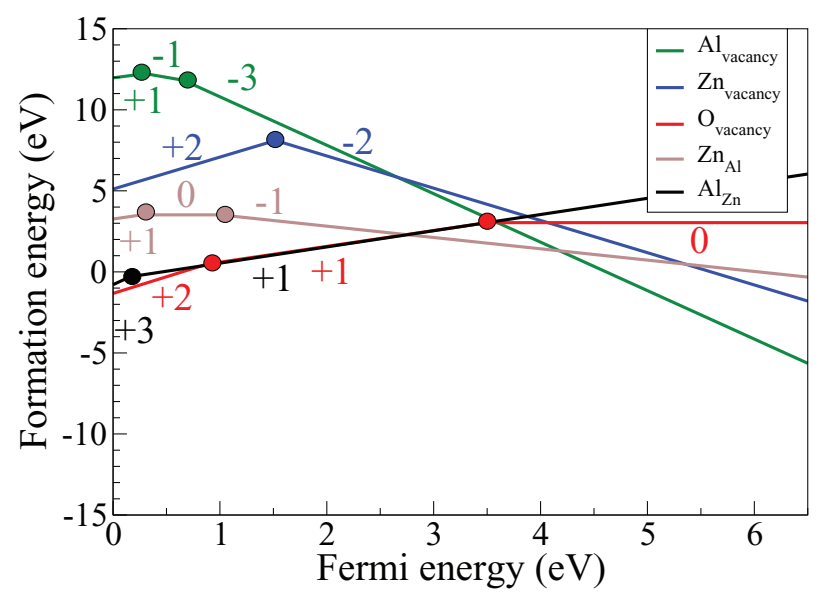

$\mathrm{O}$ - rich (Hybrid functional)

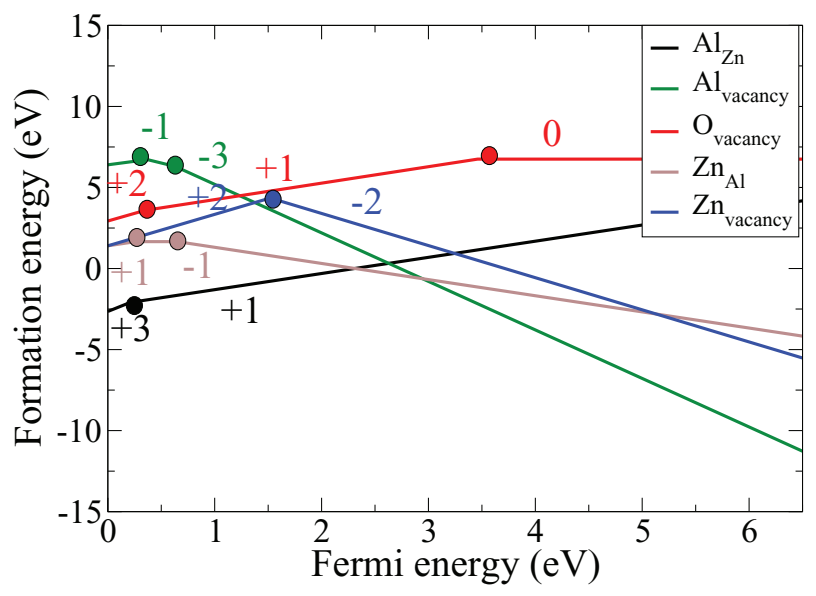

FIG. 6. (Color online) The same as Fig. 5, but obtained with the HSE06 functional. 
the increased formation energy in this corrected calculation, the amphoteric native defects can become killer defects, trapping the charge carriers originating from the shallow donor $\mathrm{Al}_{\mathrm{Zn}}$. Therefore, under equilibrium conditions, we can expect that in $\mathrm{ZnAl}_{2} \mathrm{O}_{4}$ spinel Fermi level pinning will occur in the band gap. This limits of course severely the possible application of $\mathrm{ZnAl}_{2} \mathrm{O}_{4}$ as an $n$-type transparent conducting oxide. It was also argued that the strong relaxation of the $\mathrm{Zn}_{\mathrm{Al}}$ defect could be responsible for the difference in formation energy compared to $\mathrm{Al}_{\mathrm{Zn}}$. These corrected results show that this argument does not hold and thus that one cannot only rely on the amount of relaxation between two different defects to understand their stability.

The authors acknowledge D. Scanlon (University College London) for pointing out the error. 